\title{
Identification and Characterization of the Soils of Paschimi Nayyar River Watershed in Lesser-Himalayan Region of India
}

\author{
S.K. Mahapatra ${ }^{1 *}$, Ritu Nagdev ${ }^{1}$, R.P. Yadav ${ }^{1}$ and S.K. Singh ${ }^{2}$ \\ ${ }^{1}$ ICAR-National Bureau of Soil Survey and Land Use Planning, Regional Centre, IARI \\ Campus, New Delhi-110012, India \\ ${ }^{2}$ ICAR-National Bureau of Soil Survey and Land Use Planning, Amravati Road, \\ Nagpur-440033, Maharashtra, India \\ *Corresponding author
}

\begin{tabular}{|l|}
\hline K e y w o r d s \\
Himalayan ecosystem, \\
Pauri Garhwal, \\
$\begin{array}{l}\text { Watershed, } \\
\text { Physiographic land } \\
\text { units, Land evaluation, } \\
\text { Land degradation }\end{array}$ \\
\hline Article Info \\
\hline $\begin{array}{l}\text { Accepted: } \\
\text { 17 January } 2018 \\
\text { Available Online: } \\
\text { 10 February } 2018\end{array}$ \\
\hline
\end{tabular}

\section{A B S T R A C T}

Soils of Paschimi Nayyar river watershed in Pauri Garhwal district of Uttarakhand, India were identified and characterized using remote sensing and GIS techniques. They area falls in warm humid Himalayan ecosystem. On the basis of interpreted remote sensing image data and field checks four major physiographies viz., escarpments, hill/ridge tops, side/reposed slopes and valleys were identified which were further sub divided into 8 physiographic units based on image elements and slope functions. The physiographic land units map (PLU) was generated by integrating the physiographic units and land use. Based on inventory of soil resources (field study and characterisation) 8 types of soils (P1 to P8) were identified in different PLUs of the watershed. Soils of hill/ridge tops (P1 to P3) occur on steep to very steep slopes and are mostly shallow to very shallowin depth, gravelly loamy sand to gravelly sandy loam in texture and have A-C and A-AC-C horizons. They are classified as sandy skeletal and loamy skeletal Lithic Udorthents and rated as Class IV lands. Soils of side/reposed slopes (P4 to P6) occur on moderately steep to very steep slopes and are mainly extremely shallow to moderately shallow in depth, gravelly loamy sand, gravelly sandy loam and sandy loam in texture and have A, A-C, A-AC-C horizons with patches of A-B-C horizons in lower slopes. They are classified under sandy skeletal and loamy skeletal Lithic/Typic Udorthents and coarse loamy Typic Dystrudepts and rated as class IV lands. Soils of valleys (P7 and P8) occur on gentle to moderate slopes and are comparatively well developed. They are moderately deep to very deep in depth, sandy loam to loam and clay loam in texture and have A-B-C horizons, being classified as Typic Dystrudepts and Dystric Eutrudepts and rated under class II and III lands. The study revealed that the area suffers from various kinds of soil and land degradations and hence, appropriate soil and water conservation measures are to be undertaken to arrest further losses, maintain soil health and sustainable productivity. 


\section{Introduction}

Soil is one of the precious natural resources and knowledge of its properties, occurrence, extent, distribution, constraints and potentials is essential for making any developmental planning of an area (Mahapatra et al., 2005). Soil is the most important parameter of agrienvironment which should be managed effectively and judiciously. But its multipurpose use and continuous exploitation have serious impacts on the ecology (Blum 1997; Gorai et al., 2013). The development of hills and mountainous region vis a vis protection of their ecology have become matters of concern in recent years. These areas are different from the plains in relation to topography, elevation, physiographic features, diversity of habitats for flora and fauna, ethnic diversity, land use systems and socio-economic conditions. Researchers had earlier worked on specific sites for very selective purposes (Kumar and Sharma 1987; Divakar et al., 1989; Rawat et al., 1994; Singh and Bhatnagar 1997; Ghosh and Singh 2002) but the information is still scanty especially in hilly areas like Uttarakhand of Garhwal region. Soils of this region are very prone to degradation and pose a serious threat to agricultural productivity. Inappropriate agricultural practices, overgrazing and indiscriminate deforestation are the major factors which lead to soil degradation, decline in soil fertility, crop productivity and soil quality besides environmental hazards.

The watershed management is an important concept for sustainable development of mountainous region. It is a technique of managing man made activities in a particular area by identifying the inter-relationship among landform, land use, soil and water as well as linkage between uplands and adjacent lowlands (Biswas 1987; Mishra and Ghosh 1995; Pai et al., 2007). State level information of soils may be available (Singh et al., 2004) but those kinds of data cannot provide detailed information about soils. The information about constraints and potential of each soils (soil series) can be available if those are studied at watershed/village/farm level surveys and can be used for any kind of developmental planning particularly for agricultural activities (Nagdev et al., 2017a, 2017b). Hence, a study has been undertaken to identify and characterize the soils of Paschimi Nayyar river watershed in Pauri Garhwal district of Uttarakhand, India by using the remote sensing and GIS techniques to enhance agricultural productivity.

\section{Materials and Methods}

\section{Study area}

The study area is a watershed in the Pauri Garhwal District of Uttarakhand occupying 9248 ha area extending from $30^{\circ} 00^{\prime}$ to $30^{\circ} 07^{\prime}$ $\mathrm{N}$ latitudes and $78^{\circ} 44^{\prime}$ to $78^{\circ} 50^{\prime} \mathrm{E}$ longitudes. The watershed belongs to warm humid lesser Himalayas (Agro-ecological sub region 14.2) with undulating terrains having steep slopes and ridges. The elevation ranges from 6001150mabove MSL. The climate is warm humid and annual rainfall ranges from 1000 to $1300 \mathrm{~mm}$. The soil moisture regime is udic and the temperature regime is thermic. Three steps viz., image interpretation, ground truth verification and laboratory analysis were applied to assess the soil resources.

\section{Image interpretation and field checking}

Indian Remote Sensing satellite (IRS-ID LISS III) data FCC encoded imageries of bands 2, 3 and 4 was interpreted to delineate the landform units. Main image elements viz., shape, tone or colour, pattern, shadow, association and texture were used to interpret the geomorphic features (Sahu et al., 2016). Physiography map was generated from the landform map using the slope gradient, land 
use and other terrain features of the study area. It was followed by ground truth verification to confirm the physiographic units. The boundaries were verified and corrected wherever found necessary. Present land use systems of the study area as well as geology/parent materials were observed during field study. Physiographic land unit (PLU) map was prepared by integrating physiographic units and land use systems and verified through field checks. This PLU map was used for conducting soil survey.

\section{Inventory of soil resources}

Detailed soil survey was conducted by studying minipits and master profiles in different physiographic land units and morphological characteristics were noted (Sehgal et al., 1987; Bhattacharya et al., 2009). Soil samples of each horizon of representative pedons were collected and characterised for important physico-chemical properties (Black 1965; Jackson 1966; Sharma et al., 1987).Soils were classified as per soil taxonomy (Soil Survey Staff 2014). Soil and thematic maps were generated using GIS technique. Soils were evaluated for different land capability classes (LCC) following standard techniques (Sys, 1985; Sehgal, 1996). Environmental degradational features prevailing in the watershed area were noted during field study.

\section{Results and Discussion}

Generation of physiographic land units (PLU)

Four major physiographies have been identified viz., escarpments, hill/ridge tops, side/reposed slopes and valleys (Table 1). Based on the image elements and slope functions they were further sub-divided into eight physiographic units viz., very steeply sloping escarpments, very steeply sloping hill/ridge tops, steeply sloping hill/ridge tops, very steeply sloping side/reposed slopes, steeply sloping side/reposed slopes, moderately steeply sloping side/reposed slopes, moderately sloping valleys and gently sloping valleys. Different land use systems identified in the area are barren and rocky land, scrub land, thin forest, grazing land, cultivated land, cultivated land along with plantations, respectively (Table 1). The physiographic land unit map generated by integrating physiographic units and land use has been depicted in figure 1 along with legend containing eleven PLUs. Soil resource inventory was undertaken using this PLU map wherein soils occurring in each PLU were studied for their morphological properties.

\section{Soils and their characteristics}

On the basis of ground truth study and soil resource characterization eight types of soil (P1 to P8) have been identified. The soil map has been depicted in figure 2 and their characteristics have been described in table 2 . P1 soils occur on very steeply sloping hill/ridge tops and are very shallow in depth with only $\mathrm{A}$ and $\mathrm{C}$ horizons having clear and abrupt smooth boundaries, yellowish brown to light yellowish brown in colour, loamy sand in texture with strong coarse gravels, single grain in structure, underlain by indurated bedrock at $22 \mathrm{~cm}$ depth and developed on mica schist. P2 soils occur on very steeply sloping hill/ridge tops and are shallow in depth with A-AC-C horizons having clear and abrupt smooth boundaries, dark yellowish brown to yellowish brown in colour, sandy loam in texture with strong coarse gravels, massive in structure underlain by indurated bedrock at $43 \mathrm{~cm}$ depth and developed on sandstone. P3 soils occur on steeply sloping hill/ridge tops and are shallow in depth with A-AC-C horizons having clear and abrupt smooth boundaries underlain by indurated bedrock at $35 \mathrm{~cm}$ depth and developed on mica schist. They are yellowish 
brown to light yellowish brown in colour, loamy sand in texture with strong coarse gravels and single grain in structure. P4 soils occur on very steeply sloping side/reposed slopes and are extremely shallow in depth with only A horizon having abrupt smooth boundary underlain by indurated bedrock at 10 cm depth and developed on mica schist. They are yellowish brown in colour, loamy sand in texture with strong coarse gravels and single grain in structure. P5 soils occur on steeply sloping side/reposed slopes and are moderately shallow in depth with A-AC-C horizons having clear and abrupt smooth boundaries, dark yellowish brown to yellowish brown in colour, sandy loam in texture with strong coarse gravels, massive in structure, underlain by indurated bedrock at $60 \mathrm{~cm}$ depth and developed on sandstone. P6 soils occur on moderately steeply sloping side/reposed slopes and are moderately deep in depth with A-B-C horizons having clear, gradual and abrupt smooth boundaries, yellowish brown to brown in colour, sandy loam in texture with moderate coarse gravels, disturbed and sub angular blocky in structure underlain by unconsolidated bedrock at $85 \mathrm{~cm}$ depth and developed on sandstone. P7 soils occur on moderately sloping valley terraces and are deep in depth with A-B-C horizons having clear, gradual and abrupt smooth boundaries and developed on colluvium/alluvium. They are dark yellowish brown to brown in colour, loam in texture with medium coarse gravels and disturbed and sub angular blocky in structure. P8 soils occur on gently sloping valley terraces and are very deep in depth with A-B-C horizons having clear, gradual and clear smooth boundaries and developed on alluvium/ colluvium. They are brown to dark brown in colour, loam to clay loam in texture, disturbed and sub angular blocky in structure. Thus, the study reveals that formation of soils and their properties depend mainly on landform (physiography) and the parent material.

\section{Classification of Soils}

Soils of the watershed has been classified as per USDA soil taxonomy and presented in table 3. The temperature and moisture regimes of the study area are thermic and udic, respectively. The mineralogy of all the soils are mixed type. P1soils are very shallow, excessively drained, loamy sand in texture with coarse gravels throughout the profile and have only $\mathrm{A}$ and $\mathrm{C}$ horizons underlain by indurated bedrock at $22 \mathrm{~cm}$ depth. Hence, they belong to Entisols having sandy skeletal family textural class (as the texture is loamy sand with more than 35\% coarse gravels) and are classified as very shallow, mixed, thermic, sandy skeletal Lithic Udorthents. P2 soils are shallow, excessively drained, sandy loam in texture with coarse gravels throughout the profile and have A-AC-C horizons underlain by indurated bedrock at $43 \mathrm{~cm}$ depth. Hence, they belong to Entisols having loamy skeletal family textural class (as the texture is sandy loam with more than $35 \%$ coarse gravels) and are classified as shallow, mixed, thermic, loamy skeletal Lithic Udorthents. P3 soils are shallow, excessively drained, loamy sand in texture and having coarse gravels and have AAC-C horizons underlain by indurated bedrock at $35 \mathrm{~cm}$ depth. Hence, they belong to Entisols having sandy skeletal family textural class and are classified as shallow, mixed, thermic, sandy skeletal Lithic Udorthents. P4soils are extremely shallow, excessively drained, loamy sand in texture with strong coarse gravels and have only A horizon underlain by indurated bedrock at $10 \mathrm{~cm}$ depth. Hence, they belong to Entisols having sandy skeletal family textural class and are classified as extremely shallow, mixed, thermic, sandy skeletal Lithic Udorthents. P5 soils are moderately shallow, excessively drained, sandy loam in texture with strong coarse gravels and have only A-AC-C horizons underlain by indurated bedrock at 60 cm depth. 


\section{Table.1 Interpretation of remote sensing data}

\begin{tabular}{|c|c|c|c|c|}
\hline $\begin{array}{l}\text { Map } \\
\text { Unit }\end{array}$ & Image characteristics & Physiography & Slope & Land Use \\
\hline 1 & $\begin{array}{l}\text { Medium brown to dark grayish and mottled } \\
\text { colour with coarse to rough texture and small, } \\
\text { irregular and scattered grains }\end{array}$ & Escarpment & Very steep & $\begin{array}{l}\text { Barren, } \\
\text { rocky land }\end{array}$ \\
\hline 2.1 & $\begin{array}{l}\text { Dark brown and mottled colour with coarse } \\
\text { texture and small, irregular and scattered } \\
\text { grains }\end{array}$ & Hill/ridge top & Very steep & $\begin{array}{l}\text { Barren, } \\
\text { scrub land }\end{array}$ \\
\hline 2.2 & $\begin{array}{l}\text { Brownish and mottled colour with medium to } \\
\text { coarse texture and small, irregular and } \\
\text { scattered grains }\end{array}$ & Hill/ridge top & Steep & $\begin{array}{l}\text { Scrubs, thin } \\
\text { forest }\end{array}$ \\
\hline 3.1 & $\begin{array}{l}\text { Dark brown and coarse mottled colour with } \\
\text { smooth to medium coarse texture and fine, } \\
\text { scattered grains }\end{array}$ & $\begin{array}{l}\text { Side/reposed } \\
\text { slope }\end{array}$ & Very steep & $\begin{array}{l}\text { Barren, } \\
\text { Scrubs land }\end{array}$ \\
\hline 3.2 & $\begin{array}{l}\text { Reddish and medium brown colour with } \\
\text { mottled and medium smooth texture and } \\
\text { medium, irregular and scattered grains }\end{array}$ & $\begin{array}{l}\text { Side/reposed } \\
\text { slope }\end{array}$ & Steep & $\begin{array}{l}\text { Thin forest, } \\
\text { grazing land }\end{array}$ \\
\hline 3.3 & $\begin{array}{l}\text { Medium, gray to light brown colour with } \\
\text { medium, smooth and light coloured mottlings }\end{array}$ & $\begin{array}{l}\text { Side/reposed } \\
\text { slope }\end{array}$ & $\begin{array}{l}\text { Moderatel } \\
\text { y steep }\end{array}$ & $\begin{array}{l}\text { Thin forest, } \\
\text { cultivation }\end{array}$ \\
\hline 4.1 & $\begin{array}{l}\text { Yellowish to light green colour with smooth, } \\
\text { fine mottled texture and irregular, fine and } \\
\text { scattered grains }\end{array}$ & Valley & Moderate & $\begin{array}{l}\text { Cultivation, } \\
\text { fruit } \\
\text { plantation }\end{array}$ \\
\hline 4.2 & $\begin{array}{l}\text { Light bluish tone with scattered white patches } \\
\text { and diffused, irregular grains }\end{array}$ & Valley & Gentle & Cultivation \\
\hline
\end{tabular}

\begin{tabular}{|c|c|c|c|c|c|c|c|}
\hline \multicolumn{8}{|c|}{ Table.2 Characteristics of soils } \\
\hline Soils & Horizon & Depth & Boundary & Colour (moist) & Texture & Structure* & $\begin{array}{l}\text { Coarse } \\
\text { gravels } \\
(>2 \mathrm{~mm})\end{array}$ \\
\hline \multirow[t]{3}{*}{ P1 } & A & $0-12$ & Clear, smooth & $\begin{array}{l}\text { Yellowish } \\
\text { brown }\end{array}$ & $\begin{array}{l}\text { Loamy } \\
\text { sand }\end{array}$ & $\mathrm{sg}$ & 40 \\
\hline & $\mathrm{C}$ & $12-22$ & $\begin{array}{l}\text { Abrupt, } \\
\text { smooth }\end{array}$ & $\begin{array}{l}\text { Light yellowish } \\
\text { brown }\end{array}$ & $\begin{array}{l}\text { Loamy } \\
\text { sand }\end{array}$ & sg & 45 \\
\hline & $\mathrm{R}$ & $22+$ & $\begin{array}{l}\text { Indurated } \\
\text { bedrock } \\
\text { (mica schist) }\end{array}$ & - & - & - & - \\
\hline \multirow[t]{4}{*}{$\mathbf{P 2}$} & A & $0-14$ & Clear, smooth & $\begin{array}{l}\text { Dark yellowish } \\
\text { brown }\end{array}$ & $\begin{array}{l}\text { Sandy } \\
\text { loam }\end{array}$ & $\mathrm{m}$ & 36 \\
\hline & $\mathrm{AC}$ & $14-27$ & Clear, smooth & $\begin{array}{l}\text { Yellowish } \\
\text { brown }\end{array}$ & $\begin{array}{l}\text { Sandy } \\
\text { loam }\end{array}$ & $\mathrm{m}$ & 42 \\
\hline & $\mathrm{C}$ & $27-43$ & $\begin{array}{l}\text { Abrupt, } \\
\text { smooth }\end{array}$ & $\begin{array}{l}\text { Yellowish } \\
\text { brown }\end{array}$ & $\begin{array}{l}\text { Sandy } \\
\text { loam }\end{array}$ & $\mathrm{m}$ & 50 \\
\hline & $\mathrm{R}$ & $43+$ & Indurated & - & - & - & - \\
\hline
\end{tabular}




\begin{tabular}{|c|c|c|c|c|c|c|c|}
\hline & & & $\begin{array}{l}\text { bedrock } \\
\text { (sandstone) }\end{array}$ & & & & \\
\hline \multirow[t]{4}{*}{$\mathbf{P 3}$} & A & $0-12$ & Clear, smooth & $\begin{array}{l}\text { Yellowish } \\
\text { brown }\end{array}$ & $\begin{array}{l}\text { Loamy } \\
\text { sand }\end{array}$ & $\mathrm{sg}$ & 44 \\
\hline & $\mathrm{AC}$ & $12-22$ & Clear, smooth & $\begin{array}{l}\text { Light yellowish } \\
\text { brown }\end{array}$ & $\begin{array}{l}\text { Loamy } \\
\text { sand }\end{array}$ & $\mathrm{sg}$ & 48 \\
\hline & $\mathrm{C}$ & $22-35$ & $\begin{array}{l}\text { Abrupt, } \\
\text { smooth }\end{array}$ & $\begin{array}{l}\text { Light yellowish } \\
\text { brown }\end{array}$ & $\begin{array}{l}\text { Loamy } \\
\text { sand }\end{array}$ & $\mathrm{sg}$ & 53 \\
\hline & $\mathrm{R}$ & $35+$ & $\begin{array}{l}\text { Indurated } \\
\text { bedrock } \\
\text { (mica schist) }\end{array}$ & - & - & - & - \\
\hline \multirow[t]{2}{*}{ P4 } & A & $0-10$ & $\begin{array}{l}\text { Abrupt, } \\
\text { smooth }\end{array}$ & $\begin{array}{l}\text { Yellowish } \\
\text { brown }\end{array}$ & $\begin{array}{l}\text { Loamy } \\
\text { sand }\end{array}$ & $\mathrm{sg}$ & 45 \\
\hline & $\mathrm{R}$ & $10+$ & $\begin{array}{l}\text { Indurated } \\
\text { bedrock } \\
\text { (mica schist) }\end{array}$ & 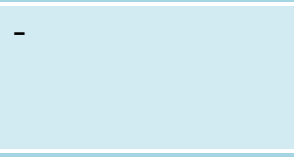 & - & - & - \\
\hline \multirow[t]{5}{*}{ P5 } & A1 & $0-12$ & Clear, smooth & $\begin{array}{l}\text { Dark yellowish } \\
\text { brown }\end{array}$ & $\begin{array}{l}\text { Sandy } \\
\text { loam }\end{array}$ & $\mathrm{m}$ & 35 \\
\hline & A 2 & $12-26$ & Clear, smooth & $\begin{array}{l}\text { Yellowish } \\
\text { brown }\end{array}$ & $\begin{array}{l}\text { Sandy } \\
\text { loam }\end{array}$ & $\mathrm{m}$ & 44 \\
\hline & $\mathrm{AC}$ & $26-45$ & Clear, smooth & $\begin{array}{l}\text { Yellowish } \\
\text { brown }\end{array}$ & $\begin{array}{l}\text { Sandy } \\
\text { loam }\end{array}$ & $\mathrm{m}$ & 46 \\
\hline & $\mathrm{C}$ & $45-60$ & $\begin{array}{l}\text { Abrupt, } \\
\text { smooth }\end{array}$ & $\begin{array}{l}\text { Yellowish } \\
\text { brown }\end{array}$ & $\begin{array}{l}\text { Sandy } \\
\text { loam }\end{array}$ & $\mathrm{m}$ & 56 \\
\hline & $\mathrm{R}$ & $60+$ & $\begin{array}{l}\text { Indurated } \\
\text { bedrock } \\
\text { (sandstone) }\end{array}$ & - & - & - & - \\
\hline \multirow[t]{6}{*}{ P6 } & AP & $0-15$ & Clear, smooth & $\begin{array}{l}\text { Dark yellowish } \\
\text { brown }\end{array}$ & $\begin{array}{l}\text { Sandy } \\
\text { loam }\end{array}$ & d & 18 \\
\hline & A12 & $15-28$ & Clear, smooth & $\begin{array}{l}\text { Yellowish } \\
\text { brown }\end{array}$ & $\begin{array}{l}\text { Sandy } \\
\text { loam }\end{array}$ & f1sbk & 20 \\
\hline & Bw1 & $28-47$ & $\begin{array}{l}\text { Gradual, } \\
\text { smooth }\end{array}$ & Brown & $\begin{array}{l}\text { Sandy } \\
\text { loam }\end{array}$ & $\mathrm{m} 1 \mathrm{sbk}$ & 22 \\
\hline & Bw2 & $47-67$ & Clear, smooth & Brown & $\begin{array}{l}\text { Sandy } \\
\text { loam }\end{array}$ & $\mathrm{m} 1 \mathrm{sbk}$ & 25 \\
\hline & $\mathrm{C}$ & $67-85$ & $\begin{array}{l}\text { Abrupt, } \\
\text { smooth }\end{array}$ & $\begin{array}{l}\text { Yellowish } \\
\text { brown }\end{array}$ & $\begin{array}{l}\text { Sandy } \\
\text { loam }\end{array}$ & f1sbk & 42 \\
\hline & $\mathrm{Cr}$ & $85+$ & $\begin{array}{l}\text { Unconsolidate } \\
\text { d bedrock } \\
\text { (sandstone) }\end{array}$ & - & - & - & - \\
\hline \multirow[t]{3}{*}{ P7 } & AP & $0-12$ & Clear, smooth & $\begin{array}{l}\text { Dark yellowish } \\
\text { brown }\end{array}$ & $\begin{array}{l}\text { Sandy } \\
\text { loam }\end{array}$ & d & 16 \\
\hline & A12 & $12-24$ & Clear, smooth & Brown & Loam & f1sbk & 18 \\
\hline & Bw1 & $24-40$ & $\begin{array}{l}\text { Gradual, } \\
\text { smooth }\end{array}$ & Brown & Loam & $\mathrm{m} 1 \mathrm{sbk}$ & 18 \\
\hline
\end{tabular}




\begin{tabular}{|c|c|c|c|c|c|c|c|}
\hline & Bw2 & $40-57$ & $\begin{array}{l}\text { Gradual, } \\
\text { smooth }\end{array}$ & Brown & Loam & m1sbk & 20 \\
\hline & Bw3 & $57-80$ & Clear, smooth & Brown & Loam & m1sbk & 22 \\
\hline & $\mathrm{C}$ & $80-105$ & $\begin{array}{l}\text { Abrupt, } \\
\text { smooth }\end{array}$ & $\begin{array}{l}\text { Dark yellowish } \\
\text { brown }\end{array}$ & $\begin{array}{l}\text { Sandy } \\
\text { loam }\end{array}$ & f1sbk & 30 \\
\hline & $\mathrm{Cr}$ & $105+$ & $\begin{array}{l}\text { Stones and } \\
\text { boulders }\end{array}$ & - & - & - & - \\
\hline P8 & AP & $0-17$ & Clear, smooth & Brown & Loam & d & 10 \\
\hline & Bw1 & $17-32$ & $\begin{array}{l}\text { Gradual, } \\
\text { smooth }\end{array}$ & Brown & Loam & m1sbk & 12 \\
\hline & Bw2 & $32-53$ & $\begin{array}{l}\text { Gradual, } \\
\text { smooth }\end{array}$ & Dark brown & $\begin{array}{l}\text { Clay } \\
\text { loam }\end{array}$ & m1sbk & 12 \\
\hline & Bw3 & $53-76$ & $\begin{array}{l}\text { Gradual, } \\
\text { smooth }\end{array}$ & Dark brown & $\begin{array}{l}\text { Clay } \\
\text { loam }\end{array}$ & $\mathrm{m} 2 \mathrm{sbk}$ & 14 \\
\hline & $\mathrm{Bw} 4$ & 76-101 & $\begin{array}{l}\text { Gradual, } \\
\text { smooth }\end{array}$ & Dark brown & $\begin{array}{l}\text { Clay } \\
\text { loam }\end{array}$ & $\mathrm{m} 2 \mathrm{sbk}$ & 14 \\
\hline & Bw5 & $101-128$ & Clear, smooth & Dark brown & $\begin{array}{l}\text { Clay } \\
\text { loam }\end{array}$ & m1sbk & 18 \\
\hline & $\mathrm{C}$ & $\begin{array}{l}128- \\
150+\end{array}$ & - & Brown & Loam & - & 20 \\
\hline
\end{tabular}

* sg: single grain;m: massive; d: disturbed; f 1 sbk: fine weak subangular blocky; $\mathrm{m} 1$ sbk: medium weak subangular blocky; $\mathrm{m} 2$ sbk: medium moderate subangular blocky

\section{Table.3 Taxonomy of soils}

\begin{tabular}{|c|c|c|}
\hline Soil & Description & Taxonomy \\
\hline P1 & $\begin{array}{l}\text { Very shallow, excessively drained, gravelly loamy sand } \\
\text { soils of yellowish brown colour developed on mica } \\
\text { schist. }\end{array}$ & $\begin{array}{l}\text { Very shallow, mixed, thermic, } \\
\text { sandy skeletal, Lithic Udorthents }\end{array}$ \\
\hline $\mathbf{P 2}$ & $\begin{array}{l}\text { Shallow, excessively drained, gravelly sandy loam soils } \\
\text { of dark yellowish brown colour developed on sandstone. }\end{array}$ & $\begin{array}{l}\text { Shallow, mixed, thermic, loamy } \\
\text { skeletal, Lithic Udorthents }\end{array}$ \\
\hline P3 & $\begin{array}{l}\text { Shallow, excessively drained, gravelly loamy sand soils } \\
\text { of yellowish brown colour developed on mica schist. }\end{array}$ & $\begin{array}{l}\text { Shallow, mixed, thermic, sandy } \\
\text { skeletal, Lithic Udorthents }\end{array}$ \\
\hline $\mathbf{P 4}$ & $\begin{array}{l}\text { Extremely shallow, excessively drained, gravelly loamy } \\
\text { sand soils of yellowish brown colour developed on mica } \\
\text { schist. }\end{array}$ & $\begin{array}{l}\text { Extremely shallow, mixed, } \\
\text { thermic, sandy skeletal, Lithic } \\
\text { Udorthents }\end{array}$ \\
\hline P5 & $\begin{array}{l}\text { Moderately shallow, excessively drained, gravelly sandy } \\
\text { loam soils of dark yellowish brown colour developed on } \\
\text { sandstone. }\end{array}$ & $\begin{array}{l}\text { Moderately shallow, mixed, } \\
\text { thermic, loamy skeletal, Typic } \\
\text { Udorthents }\end{array}$ \\
\hline P6 & $\begin{array}{l}\text { Moderately deep, somewhat excessively drained, } \\
\text { gravelly sandy loam soils, dark yellowish brown to } \\
\text { brown in colour developed on sandstone }\end{array}$ & $\begin{array}{l}\text { Moderately deep, mixed, thermic, } \\
\text { coarse loamy, Typic Dystrudepts }\end{array}$ \\
\hline P7 & $\begin{array}{l}\text { Deep, somewhat excessively drained, loam soils of } \\
\text { brown in colour developed on colluvium/alluvium. }\end{array}$ & $\begin{array}{l}\text { Deep, mixed, thermic, fine loamy, } \\
\text { Typic Dystrudepts }\end{array}$ \\
\hline P8 & $\begin{array}{l}\text { Very deep, well drained, loam to clay loam soils of Dark } \\
\text { brown colour developed on alluvium/colluvium. }\end{array}$ & $\begin{array}{l}\text { Very deep, mixed, thermic, fine } \\
\text { loamy, Dystric Eutrudepts }\end{array}$ \\
\hline
\end{tabular}


Table.4 Land capability classes and subclasses

\begin{tabular}{|c|c|c|c|c|}
\hline S. No. & Soils & Limitations & $\begin{array}{l}\text { Land } \\
\text { capability } \\
\text { class and } \\
\text { subclass }\end{array}$ & $\begin{array}{l}\text { Management } \\
\text { recommendations }\end{array}$ \\
\hline 1 & $\mathrm{P} 1, \mathrm{P} 4$ & $\begin{array}{l}\text { Very steep slope, very to } \\
\text { extremely shallow soil } \\
\text { depth, very coarse soil } \\
\text { texture (loamy sand), } \\
\text { strong gravelliness, very } \\
\text { severe erosion and } \\
\text { droughtiness. }\end{array}$ & IV sew & $\begin{array}{l}\text { Construction of engineering } \\
\text { structures, selection of plants } \\
\text { adapted to limitation of soils } \\
\text { and climate }\end{array}$ \\
\hline 2 & P2,P3 & $\begin{array}{l}\text { Steep slope, very severe } \\
\text { erosion, shallow soil } \\
\text { depth, coarsesoil texture } \\
\text { (sandy loam), gravelliness } \\
\text { and droughtiness. }\end{array}$ & IV esw & $\begin{array}{l}\text { Construction of engineering } \\
\text { structures, adoption of soil and } \\
\text { water conservation practices, } \\
\text { selection of plants adapted to } \\
\text { limitation of soils and climate. }\end{array}$ \\
\hline 3 & P5, P6 & $\begin{array}{l}\text { Moderately steep to steep } \\
\text { slopes, severe erosion, } \\
\text { limited soil depth } \\
\text { (moderately shallow to } \\
\text { moderately } \\
\text { moderate to strong } \\
\text { gravelliness. }\end{array}$ & IV es & $\begin{array}{l}\text { Development of engineering } \\
\text { structures to arrest soil erosion, } \\
\text { INM practices involving } \\
\text { recommended doses of organic } \\
\text { and inorganic fertilizers, } \\
\text { selection of crops adapted to } \\
\text { limitation of soils and climate. }\end{array}$ \\
\hline 4 & P7 & $\begin{array}{l}\text { Moderately slopping } \\
\text { lands, moderate erosion, } \\
\text { gravelliness. }\end{array}$ & III es & $\begin{array}{l}\text { Adoption of soil and water } \\
\text { conservation measures, INM } \\
\text { practices involving } \\
\text { recommended doses of organic } \\
\text { and inorganic fertilizers, } \\
\text { selection of climatically } \\
\text { adopted cereal crops and } \\
\text { horticultural plantations. }\end{array}$ \\
\hline 5 & P8 & $\begin{array}{l}\text { Sloppy landscape, } \\
\text { moderate erosion. }\end{array}$ & II e & $\begin{array}{l}\text { Cultivation of crops adaptable } \\
\text { to the climate with proper soil } \\
\text { and water management } \\
\text { practices. }\end{array}$ \\
\hline$\overline{6}$ & $\begin{array}{l}\text { Barre } \\
\mathrm{n} \\
\text { rocky } \\
\text { land }\end{array}$ & $\begin{array}{l}\text { Very steeply sloping } \\
\text { rocky lands, very severe } \\
\text { erosion, very limited or } \\
\text { no soil development. }\end{array}$ & VIII es & $\begin{array}{l}\text { Not fit for cultivation, may be } \\
\text { used for pasture development } \\
\text { with construction of proper } \\
\text { engineering structures. }\end{array}$ \\
\hline
\end{tabular}


Fig.1 Physiographic Land Units, Paschimi Nayyar River Watershed, Pauri Garhwal District, Uttarakhand

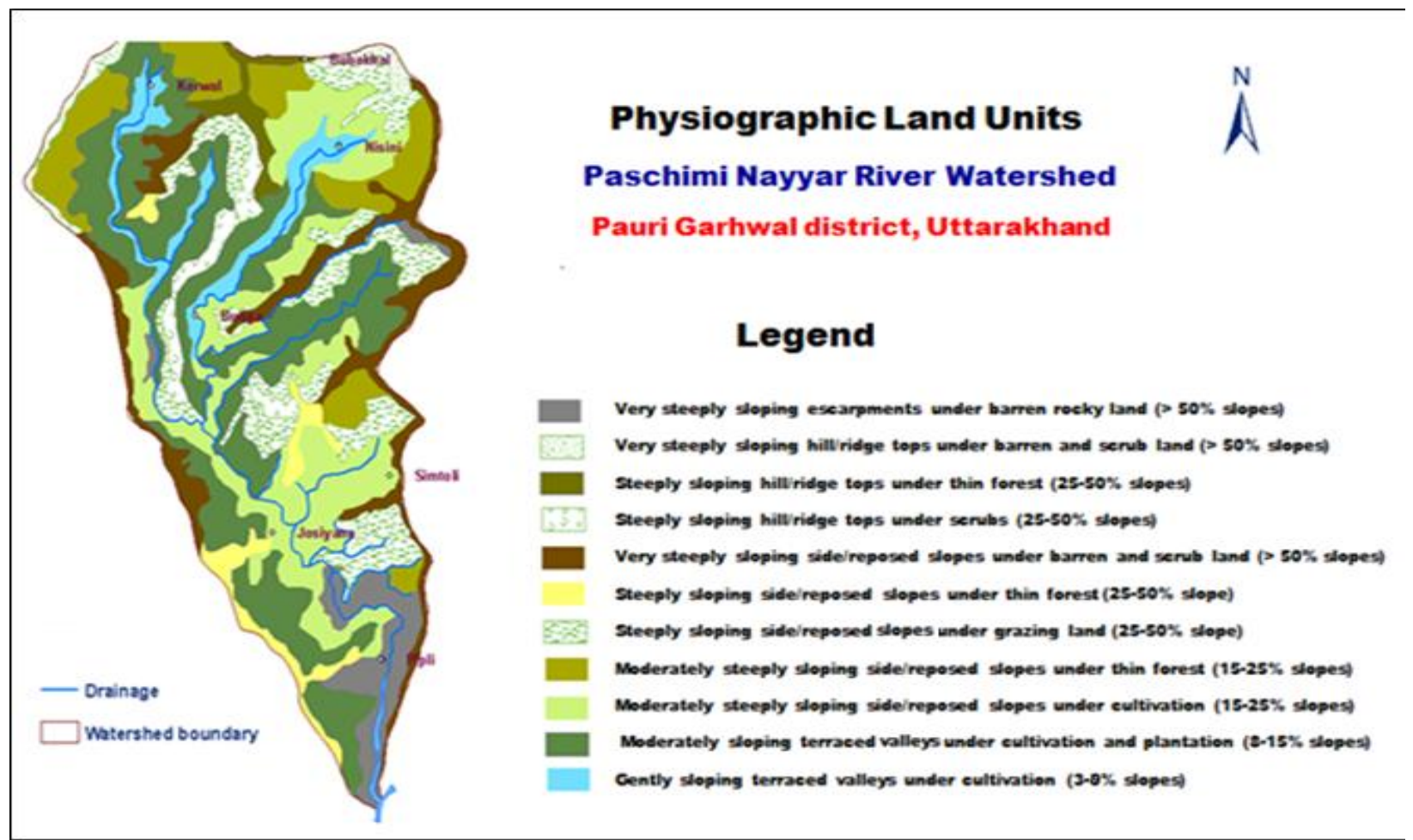

Fig.2 Soils of Paschimi Nayyar River Watershed, Pauri Garhwal District, Uttarakhand

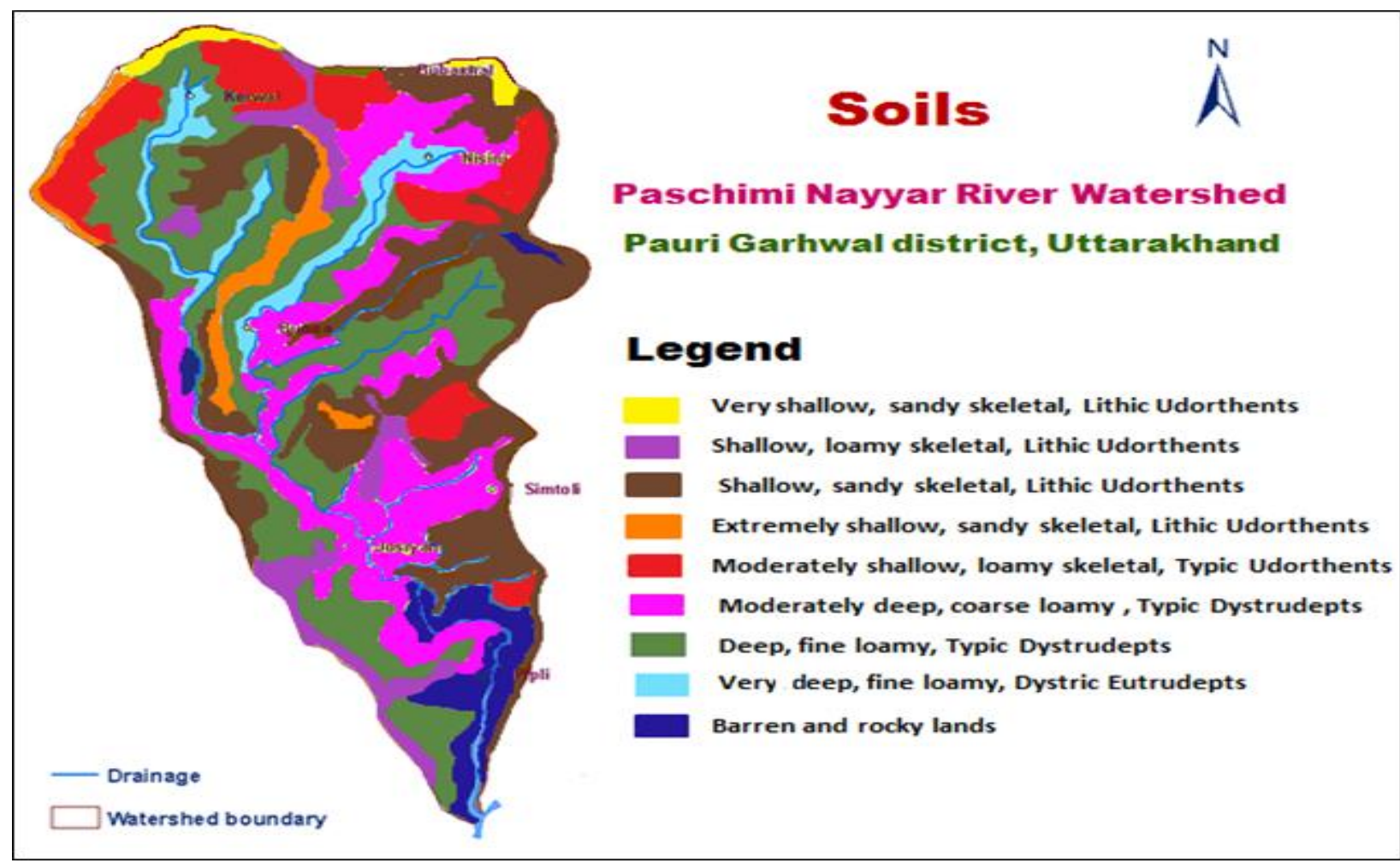


Hence, they belong to Entisols having loamy skeletal family textural class and are classified as moderately shallow, mixed, thermic, loamy skeletal Typic Udorthents. P6soils are moderately deep, somewhat excessively drained, sandy loam in texture with coarse gravels less than $35 \%$ and have A-B-C horizons underlain by unconsolidated bedrock at $85 \mathrm{~cm}$ depth. These soils are comparatively well developed having cambic diagnostic $(\mathrm{Bw})$ horizon and hence belong to Inceptisols. They have coarse loamy (clay less than $18 \%$ ) family textural class and have base saturation less than $60 \%$ and no free carbonates throughout the profile.

Hence they are classified as moderately deep, mixed, thermic, coarse loamy Typic Dystrudepts. P7 soils are deep, somewhat excessively drained, loam in texture and have A-B-C horizons. Hence, they belong to Inceptisols having fine loamy (clay more than $18 \%$ )family textural class and have base saturation less than $60 \%$ and no free carbonates throughout the profile.

Hence, they are classified as deep, mixed, thermic, fine loamy Typic Dystrudepts. P8 soils are very deep, well drained, loam to clay loam in texture and have A-B-C horizons. Hence, they belong to Inceptisols having fine loamy (clay more than 18\%) family textural class. The base saturation is more than $60 \%$ and no free carbonates throughout the profile. Therefore, these soils are classified as very deep, mixed, thermic, fine loamy Dystric Eutrudepts.

Thus, from the data it is revealed that the major soils of the watershed belong to Entisols followed by Inceptisols. Among the Entisols, Lithic (very shallow to shallow in depth i.e. less than $50 \mathrm{~cm}$ ) Udorthents cover more area than Typic Udorthents. Among the Inceptisols, Typic Dystrudepts occupy major area than Dystric Eutrudepts.

\section{Land evaluation and land use planning}

Evaluation of soil resources of the watershed is essential in order to know the potentials. Soil resource data helps in identifying the areas suitable for agriculture and other allied activities like forestry, silvi-pasture, agrihorticulture, grasslands, etc. and other commercial uses (Nagdev et al., 2017c, 2017d). The present land use of different physio graphies of the study area has been presented in table 1 . The soils of the watershed have been evaluated into land capability classes and subclasses based on their potentials, limitations as well as site characteristics viz., slope, erosion, etc. (Table 4). P1 soils occur on very steeply sloping hill/ridge tops whereas $\mathrm{P} 4$ soils occur on very steeply sloping side/reposed slopes. They are very shallow to extremely shallow in depth, very coarse textured (loamy sand) with strong gravels and prone to very severe erosion and droughtiness. Because of these limitations they have been evaluated as class-IV lands under land capability classification and subclass IV sew. They are poor in fertility status, very low in water holding capacity and productivity potential. Erosion resisting measures like construction of engineering structures are recommended in these areas besides development of pasture and forestry through selection of plants adapted to limitation of soils and climate. P2 and P3 soils occur on steeply sloping hill/ridge tops and are shallow in depth with strong gravels throughout the profile and susceptible to severe erosion and droughtiness. Hence, they are assessed as land capability class IV lands and subclass IV esw. They are poor in fertility status, low in water holding capacity and productivity potential. Construction of engineering structures and adoption of soil and water conservation practices are recommended with selection of plants suitable for pasture and forestry development as per limitation of soils and climate.P5 soils occur 
on steeply sloping side/reposed slopes whereas P6 soils occur on moderately steeply sloping side/reposed slopes. They are moderately shallow to moderately deep, coarse textured (sandy loam) with moderate to strong gravelliness and prone to severe erosion. They are evaluated as land capability class IV and subclass IVes. They are poor in fertility status, low in water holding capacity and productivity potential. Construction of engineering structures is recommended to arrest further soil degradation. Integrated nutrient management (INM) practices involving recommended doses of organic and inorganic manures may be applied and crops may be selected as per limitation of soils and climate. P7 soils occur on moderately sloping valleys and are deep, sandy loam to loam in texture having coarse gravels throughout the profile and prone to moderate erosion. They are rated as land capability class III and subclass III es. They are low to medium in fertility status, water holding capacity and productivity potential. Agricultural crops adapted to prevailing climatic conditions can be grown by adoption of soil and water conservation measures and INM practices involving recommended doses of organic and inorganic fertilizers, besides horticultural plantations. P8 soils occur on gently sloping river valleys and are very deep in depth and loam to clay loam in texture and susceptible to moderate erosion. They are assessed as land capability class II and subclass IIe. They are medium in fertility status, water holding capacity and productivity potential. These areas are recommended for cultivation of crops suitable to climatic conditions under proper soil and water management practices. The barren and rocky lands, which occur on very steep slopes and prone to very severe erosion, are not suitable for cultivation and are rated under class VIII and sub class VIIIes. However, these areas may be used for pasture development after construction of proper engineering structures.

\section{Land degradation and its remedial measures}

\section{Soil erosion}

Major areas of the watershed occur on steeply sloping hills and are prone to various types of degradations which poses threat to sustainability. The major problems are severe to very severe soil erosion and landslides. In view of these, soil and water conservation measures need to be undertaken to conserve biodiversity and sustainability. However, for adopting such remedial measures the knowledge about causes of soil erosion is prerequisite. Studies in Garhwal region (Jagat Ram et al., 2013) reveal that major causes of soil erosion are anthropogenic activities such as uncontrolled deforestation, unscientific land use, weak geological formation and active seismicity. During field survey of the study area, large deforestation activities were noticed. Very good forest had been converted into poor stock, degraded fallow lands and also unscientific agricultural activities. Besides, soils of the watershed are developed on stratified soft sedimentary and tertiary rocks which are highly prone to erosion.

\section{Conservation measures}

There is urgent need to develop site specific resource conservation technologies to arrest soil erosion and to preserve rain water, productivity potential and sustain productivity. Major conservation techniques which can be adopted are agronomic measures, mechanical measures and development of gully control structures. Agronomic practices can be adopted are up and down cultivation, ploughing, seeding and inter-culturing along the contour lines or across the slopes, etc. Inter-cropping/mixed cropping such as groundnut, green gram, soyabean, cowpea etc with maize, sorghum, castor, etc. provides sufficient ground cover 
and hence can reduce the erosion, thereby increasing productivity. Tillage is beneficial for reducing runoff and infiltration of rain water, reducing nutrient losses and enhancing crop yields. Major mechanical measures which can be adopted are contour binding, bench terracing, grassed waterways, etc. Contour bunding consisting of construction of bunds on gentle slopes are recommended for low rainfall areas. Bench terracing is the most common conservation technique in hill and mountains and can be adopted for reducing the flowing action of runoff water through construction of benches along the contours. Grassed waterways are recommended on agricultural lands to drain excess runoff water smoothly, thus reducing the erosive losses. Gully control structures are permanent structures viz., drop spillways, chute spillways and drop inlet spillways. These may be constructed for safe movement of rain water from the gully head to gully bed to reduce the erosion losses.

\section{References}

Bhattacharya, T., Sarkar, Dipak and Pal, D.K. (Ed.), 2009. Soil Survey Manual. NBSS \& LUP, Publication No. 146: 1-400.

Biswas, R. R. 1987. A soil map through lands at satellite imagery in part of the Auranga catchment in the Ranchi and Palamou districts of Bihar, India. International Journal of Remote Sensing 4: 541-543.

Black, C.A. (Ed.), 1965. Methods of Soil Analysis; Part I. American Society Madison, Wisconsis, USA.

Blum, W.E. H. 1997. Basic concepts: degradation, resilience, and rehabilitation. In Methods for assessment of soil degradation. Lal R, Blum WEH, Valentine $\mathrm{C}$ and Stewart B.A (Ed.), CRC press, New York.1-16.

Divakar, B.L., Bhagat, K.N., Tewari, J.C. and Mehta, N.S. 1989. Soil fertility status of horticultural areas in Nainital district of U.P. hills (hilly region). Progressive Horticulture, 21: 95-99.

Ghosh, B.N. and Singh, R.D. 2002.Suitability of soil test methods for available phosphorus and its critical levels for maize in acid soils of Uttaranchal hills. Journal of the Indian Society of Soil Science, 50: 132-134.

Gorai T., Ahmed, N., Mahapatra, S.K., Datta, S.C., Singh, R.D. and Sharma, R.K. 2013.Effect of topography and vegetation on soil development in Kumaon hills of North Western Himalayas. Journal of Soil and water Conservation, 12 (4): 269-276.

Jackson, M.L. 1966. Soil Chemicals Analysis. Prentice Hall of India Pvt. Ltd; New Delhi.

Jagat Ram, Singh, S.P., Yadav, R.C., Mahapatra, S.K., Sidhu, G.S., Sarkar, Dipak and Sarda, V.N. 2013.Soil Erosion in Uttarakhand. NBSS Publ. 156, NBSS \& LUP, Nagpur, pp 53.

Kumar, S. and Sharma, A.K. 1987. Numerical classification of some hill soils of Uttar Pradesh. Journal of the Indian Society of Soil Science. 35: 465-473.

Mahapatra, S.K., Jagat, Ram, Singh, S.P., Dhankar, R.P. 2005. Soil Resource Inventory of Warm Humid Kumaon Himalayas for Sustainable Land Use. Journal of the Indian Society of Soil Science, 53 (3): 390-393.

Mishra, B.B. and Ghosh, S.K. 1995. Characteriztion of soils derived from mica rich parent materials in two topo sequences. Journal of the Indian Society of Soil Science, 43 (1), 92-98.

Nagdev, Ritu, Mahapatra, S.K. and Yadav, R.P. 2017d. Assessment of AgriEnvironment in Garhwal Himalayas of India for Sustainable Productivity. International Journal of Ecology and Environmental Sciences 43 (4): 295307. 
Nagdev, Ritu, Mahapatra, S.K., Yadav, R.P. and Singh, S.K. 2017(a). Assessment of Soil Resource Potential of Warm Humid Kumaon Himalayas for Sustainable Productivity. Journal of the Indian Society of Soil Science, 65(2): 138-147.

Nagdev, Ritu, Mahapatra, S.K., Yadav, R.P. and Singh, S.K. 2017b. Delineation and Characterization of Purvi Nayyar River Watershed in Mid- Himalayan Region of India Using Remote Sensing and GIS Techniques. Int. J. Curr. Microbiol. App. Sci 6 (9): 2047-2062.

Nagdev, Ritu, Mahapatra, S.K., Yadav, R.P. and Singh, S.K. 2017c. Land capability classification and management needs in Aravalli fringes of southern Haryana for sustainable land use planning. Journal of Soil and Water Conservation, 16(2): $117-125$.

Pai, C.W., Wang, M.K. and Chiu, C.Y. 2007. Clay mineralogical characterization of a toposequence of perhumid subalpine forest soils in north eastern Taiwan. Geoderma, 138: 177-184.

Rawat, G.S., Ramana, Murthy, J., Suman, Kumar, Murthy, R.J. and Kumar, S. 1994. Characteristics and classification of soils of Shivaliks in relation to land use. Advances in Forestry Research in India, 10: 45-53.

Sahu, Nisha, Singh, S.K., Obi Reddy, G.P., Nirmal, Kumar, Nagaraju, M.S.S. and Srivastava, Rajeev, 2016. Large scale soil resource mapping using IRS P6 LISS IV and Cartosat-1 DEM in Basaltic Terrain of Central India. Journal of Indian Society of Remote Sensing, 44 (5): 811-819.

Sehgal, J. 1996. Pedology-concepts and applications. First Edition, Kalyani publishers, New Delhi.

Sehgal, J.L., Saxena, R.K. and Vadivelu, S. 1987. Field Manual; Soil Resource Mapping of Different States. Bull. 13, NBSS \& LUP, Nagpur.

Sharma, V.A.K., Krishnan, P. and Budhial, S.L. 1987. Laboratory Methods; Soil Resource, Mapping of Different States in India. Bull. 14, NBSS\&LUP, Nagpur.

Singh, R.D. and Bhatnagar, V.K. 1997. Differences in soil and leaf litter nutrient status under Pinus, Cedrus and Quercus. Indian Journal of Forestry, 20: 147-149.

Singh, S.P., Jagat Ram, Walia, C.S., Sachdev, C.B., Dhankar, R.P., Rana, K.P.C., Sehgal, J., Velayutham, M. and Gajbhiye, K.S. 2004. Soils of Uttar Pradesh for optimizing land use, NBSS Publ. 68 (Soils of India series), NBSS\&LUP, Nagpur, India, pp 1-91.

Soil Survey Staff, 2014.Keys to Soil Taxonomy, NRCS, Washington D.C., USA.

Sys, C. 1985. Land Evaluation; Part II. Agricals, Publ.7, Ghent, Belgium Pp $143-157$.

\section{How to cite this article:}

Mahapatra, S.K., Ritu Nagdev, R.P. Yadav and Singh, S.K. 2018. Identification and Characterization of the Soils of Paschimi Nayyar River Watershed in Lesser-Himalayan Region of India. Int.J.Curr.Microbiol.App.Sci. 7(02): 2185-2197. doi: https://doi.org/10.20546/ijcmas.2018.702.263 